AN. MED. INTERNA (Madrid) Vol. 19, N. ${ }^{\circ} 12$, pp. 637-639, 2002

\title{
Papel de la infección por Clostridium difficile en la reactivación de la colitis ulcerosa
}

\author{
E. VILALTA CASTEL, M. S. ALCÁZAR MONTERO*, C. NAVARRO GASPAR*, \\ M. GIMENO ARANGUEZ** \\ Servicio de Medicina Interna V. Hospital General Universitario Gregorio Marañón. \\ *Unidad de Digestivo y **Servicio de Anatomía Patológica. Cantoblanco. Madrid
}

\author{
ROLE OF CLOSTRIDIUM DIFFICILE INFECTION IN THE RELAPSE \\ OF ULCERATIVE COLITIS
}

\begin{abstract}
RESUMEN
Aportamos el caso de una mujer de edad avanzada con historia antigua de despeños diarreicos ocasionales no estudiada, que presentó un episodio de diarrea nosocomial secundaria a infección por Clostridium difficile. Se realizó una colonoscopia y biopsia ante la respuesta incompleta al tratamiento con vancomicina, siendo diagnosticada de colitis ulcerosa activa subyacente. La incidencia con la que el C.difficile se ha relacionado como desencadenate de un brote de colitis ulcerosa, se sitúa en torno al 10\%. Entre los pacientes con colitis ulcerosa es infrecuente encontrar los factores desencadenates clásicos de la infección por $C$. dif ficile: hospitalización reciente y/o uso previo de antibióticos; así como, tambien es inhabitual la visualización macroscópica de pseudomembranas. Parece existir una correlación directamente positiva entre el índice de actividad de la colitis ulcerosa y la frecuencia de la infección por C.difficile en estos pacientes. El tratamiento específico de la colitis pseudomembranosa, en la mayoría de los casos, es suficiente para controlar el brote de colitis ulcerosa, sin precisar potenciar el tratamiento de base.
\end{abstract}

PALABRAS CLAVE: Clostridium difficile. Colitis ulcerosa.

\begin{abstract}
A case of nosocomial diarrhea by Clostridium difficile in an older woman with an old history of increasing stool frecuency, is reported. Colonoscopy and biopsy was performed due to an incomplete response to vancomicyn, and the diagnosis of underlyng ulcerative colitis was made. The incidence of Clostridium difficile infection associated with the relapse of ulcerative colitis is nearly $10 \%$. In patients with ulcerative colitis, macroscopic pseudomembranes and the usual predisposing fac tors for Clostridium difficile infection, usually, are not present. It seems to exist a significant correlation between the severity of the relapse and Clostridium difficile. The specific treatment of the pseudomembranous colitis, in the majority of the cases, is sufficient for a correct control of relapse of ulcerative colitis.
\end{abstract}

KEY WORDS: Clostridium difficile. Ulcerative colitis relapse.

Vilalta Castel E, Alcázar Montero MS, Navarro Gaspar C, Gimeno Aranguez. M. Papel de la infección por Clostridium difficile en la reactivación de los Colitis ulcerosa. An Med Interna (Madrid) 2002; 19: 637-639.

\section{INTRODUCCIÓN}

La patogenia de la colitis ulcerosa $(\mathrm{CU})$ no es bien conocida. Varios autores han intentado adjudicar a determinados agentes infecciosos un papel etiológico, no habiendo sido confirmado por estudios ulteriores (1-4). No obstante, es relativamente frecuente, identificar en los días previos a la reactivación de la enfermedad, uno ó varios factores desencadenantes, entre los que cabe destacar, por su frecuencia, la infección respiratoria de vías altas o infecciones gastrointestinales por Clostridium difficile, enterovirus y citomegalovirus, entre otros (5-7). Existen varios mecanismos fisiopatológicos que potencialmente podrían predisponer al sobrecrecimiento del C. difficile en los pacientes con CU, desde la resección intestinal, hasta el papel de la sulfapiridina en la modificación de la flora intestinal $(8,9)$. Presentamos el caso de una paciente de edad avanzada, con historia previa de alteración del ritmo intestinal, que debutó con un episodio de diarrea nosocomial secundario a una colitis pseudomembranosa(CPM), siendo diagnosticada de colitis ulcerosa activa subyacente, ante la respuesta incompleta al tratamiento con vancomicina.

\section{CASO APORTADO}

Mujer de 75 años, ingresada en nuestro servicio por episodio de diarrea nosocomial. Entre sus antecedentes personales destacaba: hipertensión arterial, diabetes mellitus insulíndependiente, accidente cerebrovascular hemisférico derecho en 1997 con mínimas secuelas y trastornos del ritmo intestinal de forma esporádica de dos años de

Trabajo aceptado: 14 de septiembre de 2001

Correspondencia: E.Vilalta Castel. C/ Mira el Sol, 17,B-2.28005 Madrid. e-mail: med022829@nacom.es. 
evolución, no estudiado. Seis semanas antes de su traslado, ingresó en el Servicio de Rehabilitación por accidente cerebrovascular hemisférico izquierdo. Quince días más tarde, recibió tratamiento $\left(\mathrm{T}^{\circ}\right)$ con ciprofloxacino (500 mg cada 12 horas vía oral-v.o.- durante 5 días), por síndrome febril sin focalidad. Dos semanas después de finalizado el $\mathrm{T}^{\circ}$ antibiótico, la paciente comenzó con un cuadro de diarrea con moco, dolor abdominal y deshidratación. La exploración abdominal fue inespecífica y el tacto rectal mostró heces pastosas con presencia de moco. Se inició $\mathrm{T}^{\mathbf{o}}$ con vancomicina v.o. (125 mg/6 horas) ante la sospecha de colitis pseudomembranosa. Setenta y dos horas después, presentó cuadro de abdomen agudo, con importante dolor y distensión abdominal con ausencia de ruidos intestinales. El tacto rectal fue similar al previo. En la analítica practicada, se observó 17.200 leucocitos $/ \mathrm{mm}^{3}$ con desviación izquierda, $\mathrm{Hb} 11,1 \mathrm{~g} / \mathrm{dl}$ con volúmenes corpusculares normales, plaquetas $519.000 / \mathrm{mm}^{3}$ e hiperfibrinogenemia. La amilasemia seriada fue normal. El ECG mostró ritmo sinusal sin otras alteraciones. La radiografía simple de abdomen evidenció gran dilatación del colon con gas en recto y ausencia de niveles hidroaéreos; y, la ecografía abdominal: colelitiasis sin otros hallazgos. Se decidió $\mathrm{T}^{\mathbf{o}}$ conservador, manteniendo la vancomicina v.o., experimentando a las 72 horas evidente mejoría clínica, aunque persistía la emisión de abundante moco verdoso y en la analítica se mantenían los reactantes de fase aguda elevados (VSG: $115 \mathrm{~mm} / 1^{\mathrm{a}}$ hora y fibrinógeno $584 \mathrm{mg} / \mathrm{dl}$ ). Se recibió el resultado positivo del test para la toxina del $C$. difficile, siendo el coprocultivo anodino. Trás estabilizar a la paciente, se practicó una colonoscopia que evidenció en colon descendente y recto, una mucosa hiperémica, muy friable y erosionada. Se biopsió la mucosa lesionada, recibiéndose fijados en formol tamponado al 10\%, múltiples fragmentos entre 1-2 mm, que se incluyeron en su totalidad para estudio histológico, observándose una mucosa de tipo intestino grueso que mostraba moderado infiltrado inflamatorio crónico linfocitario en lámina propia, con atrofia de las criptas glandulares, pérdida de capacidad mucípara, distorsión de la arquitectura glandular, con cambios regenerativos epiteliales glandulares; sobre estos cambios, se observaron, signos de activación de la misma, consistentes en abscesos crípticos e infiltrado neutrofílico superficial (Fig. 1). En uno de los fragmentos, se observó necrosis de la mucosa cubierta por escara necrótica y fibrinoleucocitaria, correspondiendo a fondo de úlcera (Fig. 2). Todos estos datos configuraron el diagnóstico histológico de Enfermedad Inflamatoria Crónica Intestinal con signos de reagudización y/o inflamación aguda sobreañadida. Se mantuvo la vancomicina durante 10 días y se administraron enemas de mesalazina, siendo la evolución favorable. La toxina del C. difficile, tras finalizar el $\mathrm{T}^{\mathrm{o}}$, fue negativa. Dos meses más tarde, en revisión en consultas externas, la paciente mantenía buen estado general, dos deposiciones blandas al día sin dolor abdominal, habiéndose normalizado la analítica.

\section{DISCUSIÓN}

El Clostridium difficile, para algunos autores (10) es el patógeno más frecuente entre los pacientes con recaída de su EII , atribuible a factores infecciosos. La incidencia aproximada de la CPM en pacientes con CU varía entre el 5-24\% $(5,6)$. A diferencia de lo observado en nuestro caso, se ha descrito que los factores predisponentes más habituales para el desarrollo de la CPM: el uso previo de antibióticos y/u hospitalización reciente, no suelen estar presentes en el grupo de pacientes con CU (5); si bien, esta opinión no es sostenida por otros autores (11). Probablemente, estas diferencias puedan justificarse por la diferente procedencia de los pacientes estudiados: población hospitalizada o controlada en consultas externas. Nuestra paciente, en las semanas previas al diagnóstico, había sido tratada con ciprofloxacino y llevaba hospitalizada seis semanas en el Servicio de Rehabilitación. El mecanismo de colonización por $C$. difficile en los

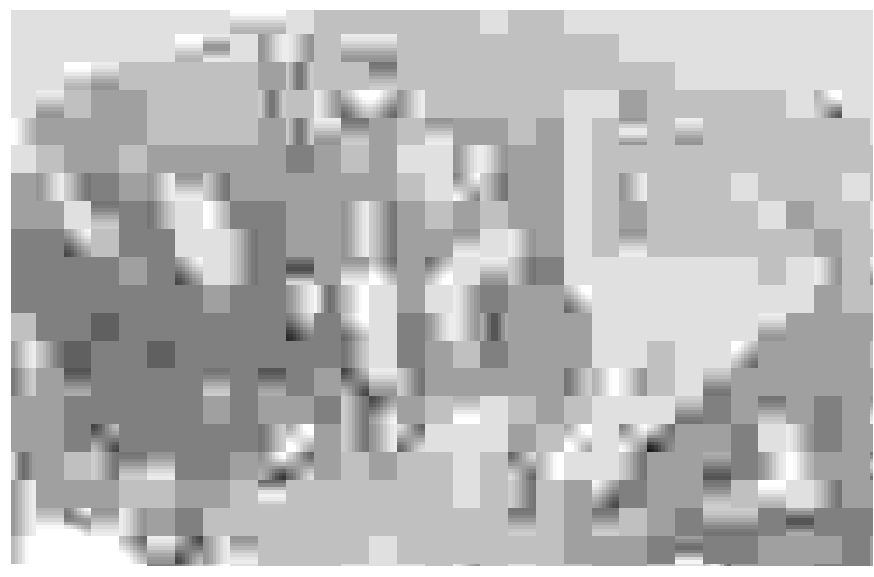

Fig. 1. Mucosa de intestino grueso que muestra de forma parcheada, moderado infiltrado inflamatorio crónico en lámina propia, atrofia gladular, distorsión de la arquitectura con cambios regenerativos epiteliares glandulares y presencia de abscesos crípticos (H-E).

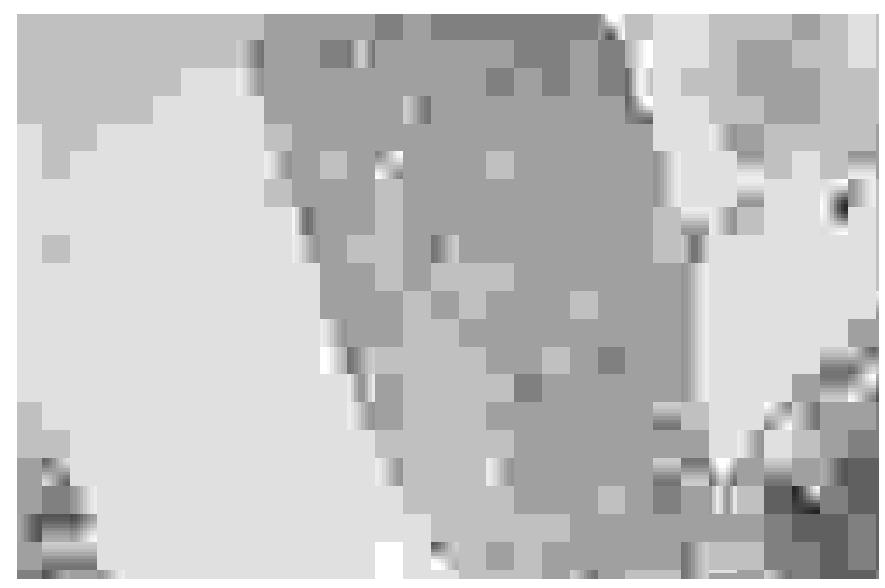

Fig. 2. Tejido necrótico y fibrino-leucocitaria de mucosa de colon descendente (H-E x 200).

pacientes no tratados previamente con antibióticos no es bien conocido. Se especula que la sulfapiridina sería capaz de modificar la flora intestinal (12); y, por lo tanto, predisponer al sobrecrecimiento del $C$. difficile. Sin embargo, otros estudios (13), demuestran que tanto la sulfasalazina como sus metabolitos, incluído el 5-ASA, inhiben in vitro el crecimiento del $C$. difficile. Paralelamente, Tremain y cols. (14), al igual que Weber y cols. (10), concluyen que la infección por $C$. difficile fue significativamente menor entre los pacientes con EII que tomaban sulfasalazina. La disparidad de estos resultados pudiera atribuirse a la falta de homogeneidad de los pacientes estudiados, al no referir sus autores otros posibles factores determinantes de la colonización por C. difficile, como la existencia previa de resección intestinal, hospitalización reciente en los pacientes ambulantes u otros tratamientos no antibióticos asociados.

Las características histológicas observadas en la biopsia de colon de nuestra paciente, muestran rasgos evidentes de EII (12), sobreañadiéndose a ellos datos de agudización: abscesos crípticos, infiltrado neutrofílico superficial y escara fibrinoleucocitaria de fondo de úlcera. Todo ello plantea varias posibilidades diagnósticas: o bien se trata de colitis ulcerosa en fase de reagudización marcada, con ulceraciones 
extensas de la mucosa; o bien, que los signos inflamatorios agudos correspondan a colitis aguda infecciosa sobreañadiada a EII. Las características clínicas descartan colitis ulcerosa fulminante, por lo que resulta más probable que se trate de la segunda posibilidad. El tejido necrótico descrito no se corresponde con los hallazgos típicos de pseudomembranas, que están característicamente formadas por moco, fibrina y leucocitos neutrófilos. En la CPM típica, cubren en forma de seta o nube la mucosa agudamente inflamada (13). De hecho, cuando el $C$. difficile complica a la $\mathrm{CU}$, es poco frecuente su visualización (4), como ocurrió en nuestro caso. Posiblemente, la mucosa inflamada de los pacientes con CU sea susceptible al daño por C.difficile a concentraciones demasiados bajas como para provocar su formación, alcanzando formas leves de CPM, desde el punto de vista histológico; así mismo, es probable que la mucosa colónica, previamente dañada por la propia CU en fase activa, no soporte su formación. En algunas series $(5,14)$ se ha establecido una correlación directa entre la CPM y el índice de actividad de la CU. La mayoría de los autores $(4,14,15)$ coinciden en que el $\mathrm{T}^{\mathrm{o}}$ específico de la CPM suele conseguir un adecuado control del brote de CU, sin pre- cisar una actitud trapéutica más agresiva. A nuestra paciente se le administró mesalazina en forma de enemas, alcanzando una rápida mejoría. Es posible que los fármacos utilizados para tratar la infección por $C$. difficile tengan un efecto terapéutico no relacionado con la EII. La utilidad del metronidazol en la enfermedad de Crohn está bien documentado $(16,17)$; no así en la CU, dónde no se ha demostrado beneficio alguno $(18,19)$, excepto con el objetivo de conseguir la erradicación del $C$. difficile. El mismo razonamiento es válido para la vancomicina en las recaídas de la CU (20). Por lo tanto, el $\mathrm{T}^{\mathrm{o}}$ específico de la infección por $C$. difficile mejora el manejo de algunas recaídas; y, evita los efectos adversos del incremento del $\mathrm{T}^{\mathrm{o}}$ de base de la CU.

Podemos concluir que la infección por $C$. difficile en los pacientes con $\mathrm{CU}$ es más frecuente que en la población general, pudiendo provocar una reactivación de la enfermedad en un limitado número de casos; no obstante, el índice de sospecha clínica debe ser elevado, puesto que en la mayoría de los casos, el $\mathrm{T}^{\mathbf{o}}$ específico de la infección por $C$. difficile va a ser capaz de controlar el cuadro clínico sin necesidad de incrementar el $\mathrm{T}^{\mathrm{o}}$ de base.

\section{Bibliografía}

1. Moses PH, Moore BR, Ferrentino N, Bensen SP, Vecchio JA. Inflammatory bowel disease. Origin, presentation and course. Postgrad Med 1998; 103 (5): 77-84

2. Brown VJ, Hudson MJ, Patrick J, Matthews CW, Hill MJ, Gent AE, et al. Search for Enteric Microbial Pathogens in Patients with Ulcerative Colitis. Digestion 1992; 53: 121-8.

3. Desreumaux P, Colombel JF. Intestinal microflora and cronic inflammatory bowel diseases. Gastroenterol Clin Biol 2001; 25 Spec No 2: C 89-93.

4. Farell RJ, LaMont JT. Microbial factors in inflammatory bowel disease. Gastroenterol Clin North Am 2002; 31: 41-62.

5. Papadakis KA, Tung JK, Binder SW, Kam LY, Abreu MT, Targan $\mathrm{SR}$, et al. Outcome of cytomegalovirus infections in patiens with inflammatory bowel disease. Am J Gastroenterol 2001; 96: 2137 42

6. Bertalot G, Villanacci V, Gramegna M, Orvieto E, Negrini R, Saleri A, et al. Evidence of Epstein-Barr virus infection in ulcerative colitis. Dig Liver Dis 2001; 33 (7): 551-8.

7. Hermens DJ, Miner Ph B Jr. Exacerbation of Ulcerative Colitis. Gastroenterology 1991; 101: 254-62.

8. Wang A, Takeshima $\mathrm{F}$, Ikeda $\mathrm{M}$, Ohnita $\mathrm{K}$, Foruso $\mathrm{H}$, Isomoto $\mathrm{H}$, et al. Ulcerative colitis complicating pseudomembranous colitis of the right colon. J Gastroenterol 2002; 37 (4): 309-12

9. García-Osogobio S, Takahashi T, Gamboa-Domínguez A, Medina H, Arch J, Mass W, et al. Toxic pseudomembranous colitis in a patient with ulcerative colitis. Inflamm Bowel Dis 2000; 6: 188-90.

10. Weber P, Koch M, Wolfgang RH, Scheulen M, Harro J, Hartnann F. Microbic Superinfection Relapse of Inflammatory Bowel Disease. J Clin Gastroenterol 1992; 14 (4): 302-8.

11. Meyers S, Mayer L, Bottone E, Desmond E and Janowitz HD. Occurrence of Clostridium difficile Toxin During the Course of Inflammatory Bowel Disease. Gastroenterology 1981; 80: 697-700.

12. Hartley MG, Hudson MJ, Swarbrick ET, Grace RH, Gent AE, Hellier MD. Sulphasalazine treatment and the colorectal mucosa-associated

flora in ulcerative colitis. Aliment Pharmacol Ther 1996; 10 (2): 157 63.

13. Sandberg-Gertzen H, Kjellander J, Sundberg-Gilla B, Jarnerot G. In vitro effects of sulfasalazine, azodisal sodium, and their metabolites on Clostridium difficile and some other faecal bacteria. Scand J Gastroenterol 1985; 20: 607-12

14. Tremaine WJ, Bille J, Huizenga KA, Washington JA, Ilstrup DM. Factors wich influence the ocurrence of Clostridium difficile infections in inflammatory bowel disease(abstr.). Gastroenterology 1983; 84: 1337.

15. G.Schumacher, B.Kullberg, B.Sandstedt. A prospective study of first attacks of Inflammatory Bowel Disease and Infectious Colitis. Histologic course during the 1st year after presentation. Scand J Gastroenterol 1994; 29: 318-32.

16. SV Nash, R.Bourgeault, M.Sands. Colonic disease associated with a positive assay for Clostridium difficile Toxin: A retrospective study. J Clin Gastroenterol 1997; 25 (2): 476-9.

17. Kochhar R, Ayyagari A, Goenka MK, Dhali GK, Aggarwal R and Mehta K. Agents in Exacerbations of Ulcerative Colitis in India. J Clin Gastroenterol 1993;16 (1): 26-30.

18. LaMont JT, Trnka Y. Therapeutic implications of Clostridium difficile toxin during relapse of chronic inflammatory bowel disease. Lancet 1980; 1: 381-3.

19. Robinson M. Medical therapy of inflammatory bowel disease for the 21st century. Eur J Surg Suppl 1998; (582): 90-8.

20. Gilat T, Suissa A, Leichtman G, delpre G, Pavlotsky M, Gossman A, et al. A comparative study of metronidazole and sulfasalazine in inactive, not severe, ulcerative colitis. J Clin Gastroenterol 1987; 9: 415-7.

21. Chapman RW, Selby WS, Jewell DP. Controlled trial of intravenous metronidazole as an adjunct to corticosteroids in severe ulcerative colitis. Gut 1986; 27 : 1210-2.

22. Dickinson RJ, O’Connor HJ, Pinder I, Hamilton I, Johnston D, Axon ATR. Doubled blind controlled trial of oral vancomycin as adjuctive treatment in acute exacerbations of idiopatic colitis. Gut 1985; 26: $1380-4$. 